\title{
A LEACH-Head Expected Frequency Appraisal Algorithm for Water-Environment Monitoring Networks*
}

\author{
Chenmin $\mathrm{Li}^{1,2}$, Guoping Tan ${ }^{1,2}$, Jingyu $\mathrm{Wu}^{1}$, Zhen Zhang ${ }^{1}$, Lizhong $\mathrm{Xu}^{1,2 \#}$ \\ ${ }^{1}$ College of Computer and Information Engineering, Hohai University, Nanjing, China \\ ${ }^{2}$ Institute of Communication and Information System, Hohai University, Nanjing, China \\ E-mail: ${ }^{\#}$ zhxu@hhu.edu.cn, zz_hhuc@163.com \\ Received July 18, 2011; revised August 12, 2011; accepted August 27, 2011
}

\begin{abstract}
Water-environment monitoring network (WMN) is a wireless sensor network based real-time system, which collects, transmits, analyzes and processes water-environment parameters in large area. Both cluster selection mechanisms and energy saving strategies play an important role on designing network routing protocols for the WMN. Since those existing routing algorithms can not be used directly in the WMN, we thus propose an improved version of LEACH, a LEACH-Head Expected Frequency Appraisal (LEACH-HEFA) algorithm, for the WMN in this paper. Simulation results show that the LEACH-HEFA can balance the energy consumption of nodes, rationalize the clustering process and prolong the network lifetime significantly in the WMN. It indicates that the LEACH-HEFA is suitable to the WMN.
\end{abstract}

Keywords: WSN, LEACH Protocol, Cluster-Head Selection, Water-Environment Monitoring

\section{Introduction}

The wireless sensor network (WSN) located in the monitoring area is comprised of the sensor nodes with the capability of sensing, storage, correspondence and computation [1,2]. Since the influence of terrain, climate and flow, the regional hydrologic information has intense mutability. They can not be real-time monitored synthetically $[3,4]$. It is necessary to introduce the newly arisen technique for the area monitoring system, and constructed the WSN based regional water-environment monitoring network. The advantages of WMN are more obvious in harsh environment and the area which human could not access [5].

At present, there are many routing protocols of WSN [6,7], such as SPIN (Sensor Protocols for Information via Negotiation), MTE (Minimum Transmission Energy), DD (Directed Diffusion) and LEACH (Low-Energy Adaptive Clustering Hierarchy) protocols etc. Based on the main network structure, the routing protocol of WSN

\footnotetext{
${ }^{*}$ This paper is supported by the National Natural Science Foundation of China (No.61001068), the Special Funds for Scientific Research on Public Causes (No. 201201118) and the "948" Project (No. 201025) of the Ministry of Water Resources of the People's Republic of China.
}

could be divided into plane routing protocol and cluster routing protocol [8]. Plane routing protocol needs to maintain a large routing table and take up numerous storage spaces. It thus is not suitable to be applied in the WMN. This problem would be solved by the cluster routing protocol to some extent. Clustering is an important energy-saving method in WSN [9].

The cluster-head selection mechanism directly affects the energy consumption and network lifetime in WSN based water-environment monitoring systems. Thus, the cluster-head selection mechanism and the energy saving strategy would be considered as the important aspects in the design of network routing protocols. However, the present routing protocols usually have problems such as previously selected cluster-head, unbalancing energy loads and short lifetime. It can not be applied to the real-time WMN with large area. In this paper, the classic clustering routing algorithm $\mathrm{LEACH}$ and $\mathrm{LEACH}-\mathrm{C}$ were analyzed. Based on the idea of cluster-head expected frequency appraisal, LEACH-HEFA (LEACH- Head Expected Frequency Appraisal) algorithm is proposed. Depending on cluster-head selection and energy balance, the algorithm can prolong the lifetime of network.

The rest of the paper is organized as follows. The first 
section presents the theory and cluster-head selection mechanism of LEACH and LEACH-C algorithms, and the problems existed in the two algorithms. The second section proposes a LEACH-HEFA algorithm based on the idea of cluster-head expected frequency appraisal, and then describes the realization procedure in detail. The simulation results are shown in the third section. The final section gives the conclusions.

\section{Analysis of Classical Algorithms}

In water-environment monitoring system, to monitor a large range with WSN, the paper designs the clustering routing algorithm based on LEACH. The reasons are as follows: 1) based on a clustering routing protocol, LEACH can satisfy the requirement of real-time transmits, analyze and process the water-environment parameters better than the traditional plane routing protocol. 2) Because of using local control technology and MAC protocol with low energy consumption, LEACH protocol is further satisfy the demand on the node energy control and network throughput. Because of the features above, the LEACH routing protocol based on the hierarchic structure can be more suitable to be applied in the large range $\mathrm{WMN}$ than other routing protocols.

\subsection{Description of Classic LEACH Algorithm}

As clustering routing algorithms proposed in WSN, LEACH (Low energy adaptive clustering hierarchy) have received lots of researchers' attention. Its thought of clusters has far-reaching influences on many important clustering routing algorithms suggested later [10]. The basic principle is that it assigns overall energy consumption of the network uniformly to each sensor node through periodically selecting different nodes as cluster-head. This makes the survival time of nodes close to the lifetime of network. Thus, the energy consumption can be reduced and the lifetime of the entire network can be prolonged [11].

The operation of LEACH is divided into several rounds. Each round begins with a set-up phase when the clusters are organized, followed by a steady-state phase, as shown in Figure 1.

In the set-up state when the clusters are organized, the LEACH sets a threshold value $T(n)$ first, and then sensor node $i$ generates a random number between 0 and 1 automatically by distributed computing. If the random number $<T(n)$, the node will become the cluster-head of the current round $r$, and common nodes join in the nearest cluster. After a period of data transmission, the network starts cluster reconstruction of the new round. And the circular processes like that.

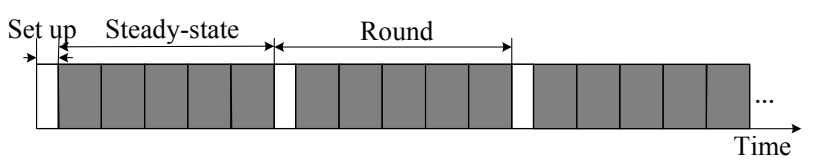

Figure 1. Time-line in LEACH operation.

The threshold value [12] is:

$$
T(n)=\left\{\begin{array}{cc}
\frac{p}{1-p \times[r \cdot \bmod (1 / p)]}, & n \in G \\
0, & \text { otherwise }
\end{array}\right.
$$

where $p$ is the probability of the nodded being selected as a cluster-head node; $r$ is the number of rounds passed, and $G$ is the collection of ordinary nodes; mod denotes modulo operator. The value of $p$ is the expected number of cluster-head nodes. In the LEACH algorithm, all nodes in the cluster take it turns to act as the head node, to achieve the purpose of balancing node energy consumption. Therefore, only the nodes that have not already been cluster-heads recently and have more energy available may become cluster-heads at round $r+1$.

Once the cluster-head is selected, all nodes join the corresponding cluster according to the broadcast signal intensity of the cluster-head node. Then, the cluster set-up phase of this round is completed. When the cluster-head assigns time slots for its members using TDMA mode, the network will enter the steady-state. In this phase, after all member nodes sent monitoring data information, the head node will process data fusion, and then send data information to the base station. After this round, it turns to the next round, and starts cluster reconstruction of the new round [13].

\subsection{Description of LEACH-C Algorithm}

Among many improved LEACH algorithms, LEACH-C (LEACH-Centralized) is the most representative algorithm [14]. In the LEACH-C algorithm, the base station is responsible for the selection of cluster-heads. It is a centralized cluster-heads algorithm [15]. After each node reports its location and current energy, the base station will select cluster-heads. According to the information of all the nodes sending, the base station will select the cluster-heads set based on appropriate number and location. Then, according to the smallest square of the distance principle between all member nodes to the cluster-head node, the base station broadcasts the cluster-head set and cluster structure.

\subsection{Existing Problems}

Although LEACH-C solves the problem of uncertainty 
on the number of cluster-head at each round in LEACH, it still has problems such as pre-selection cluster-head, equal opportunities for cluster-head selection mechanism, and the unbalancing energy loads. This phenomenon means that nodes with less energy remaining may be also become cluster-heads. However, once these nodes become cluster-head, their energy will soon exhaust. In the later periods of the network, even the phenomenon of the cluster-head is dead where it have not energy to forward the information may occurred. Therefore, the selection mechanism of cluster-head affects the performance and lifetime of the entire network. Besides, through the analysis of the node residual energy distribution, the network lifetime has direct relation to whether the energy utilization is balanced.

\section{Improved LEACH with HEFA}

In order to solve above mentioned problems, an improved LEACH algorithm, LEACH-HEFA (LEACHHeader Expect Frequency Appraisal) algorithm is proposed based on the idea of cluster-head expected frequency appraisal. The algorithm use the cluster-head expect frequency appraisal function as a parameter in the mechanism of the cluster-head selection, and add up the optimum solution of cluster-head number. If the node has higher residual energy, it will have more probability to be selected as cluster-head, which balances node energy efficacious. The algorithm also uses the concept of rounds. Each round of the cluster is also composed of set-up phases and steady-state phases. The following is the procedure of the LEACH-HEFA.

\subsection{Set-up Phase}

Now let $N$ denotes the number of nodes in the network, and $R$ denotes the number of rounds of the network until now, $\Delta t$ denotes the time spent per round, $H$ denotes the number of cluster-head. Each member node sends a Join-REQ message to cluster-head, which consist of itself ID and the current residual energy. The following is the realization procedure of set-up phase.

At time of $t+\Delta t$, the average energy consumption of the single cluster-head node is computed by:

$$
\bar{E}_{\text {head }}(t+\Delta t)=\frac{\sum_{i=1}^{H}\left[E_{i_{-} \text {current }}(t)-E_{i_{-} \text {current }}(t+\Delta t)\right]}{H}
$$

where, $E_{i_{\text {_current }}}(t)$ and $E_{i_{\text {_c current }}}(t+\Delta t)$ are the current residual energy of node $i$ at time of $t$ and $t+\Delta t$, respectively.

Then, the average energy consumption of the single member node is given by:

$$
\bar{E}_{\text {common }}(t+\Delta t)=\frac{\sum_{j=1}^{N-H}\left[E_{j_{-} \text {current }}(t)-E_{j_{-} \text {current }}(t+\Delta t)\right]}{N-H} \text { (3) }
$$

The maximum expected rounds of network operation can be calculated as:

$$
R_{\text {expect }}=\frac{\sum_{k=1}^{N} E_{k_{-} \text {current }}(t+\Delta t)}{\bar{E}_{\text {head }}(t+\Delta t)+\bar{E}_{\text {common }}(t+\Delta t)}
$$

The expected frequency appraisal factors of node $i$ becoming cluster-head satisfy the following formula:

$$
\begin{aligned}
K_{i_{-} \text {expect }} & \times \bar{E}_{\text {head }}(t+\Delta t)+\left(R_{\text {expect }}-K_{i_{\_} \text {expect }}\right) \\
& \times \bar{E}_{\text {common }}(t+\Delta t) \leq E_{i_{-} \text {current }}(t+\Delta t)
\end{aligned}
$$

From (5), we have:

$$
K_{i_{-} \text {expect }} \leq \frac{E_{i_{-} \text {current }}(t+\Delta t)-\bar{E}_{\text {common }}(t+\Delta t)}{\bar{E}_{\text {head }}(t+\Delta t)-\bar{E}_{\text {common }}(t+\Delta t)}
$$

In the time of $t+2 \Delta t, t+3 \Delta t, t+4 \Delta t \cdots$, repeat the above steps, update the $\bar{E}_{\text {head }}(t+2 \Delta t), \bar{E}_{\text {common }}(t+2 \Delta t)$, $R_{\text {expect }}$ and $K_{i \text { expect }}$ at the new round.

It is shown that, through repeat the above steps, the expected frequency appraisal factors of node $i$ becoming cluster-head changes dynamicly. As the network energy consumption, $K_{i_{-} \text {expect }}$ decreases gradually. Evidently, $K_{i \_ \text {expect }}$ of the second round is the maximum, presume as $K_{i_{-} \text {expect(max) }}$. If $K_{i_{-} \text {expect }}$ close to $K_{\left.i_{-} \text {expect( } \max \right)}$, it shows that the node has higher residual energy and more probability to be elected as cluster-head. Otherwise, if $K_{i_{-} \text {expect }}$ close to 0 , the node has little residual energy and less probability to be elected as cluster-head. Therefore, use the number of cluster-head node appraisal function $\Psi\left(K_{i_{-} \text {expect }}\right)$ as a parameter in the cluster-head selection mechanism. The function value is between $(0$, 1 ), which could optimize the cluster-head selection more effectively. Figure 2 shows the general curve of clusterhead expected frequency appraisal function $\Psi\left(K_{i_{-} \text {expect }}\right)$.



Figure 2. The curve of cluster-head expected frequency appraisal function $\boldsymbol{\Psi}\left(\boldsymbol{K}_{\text {i_expect }}\right)$. 
Clearly, the average energy consumption of the cluster-head node and common member nodes are different. In addition, with the network and iteration process, cluster-head expected frequency appraisal function $\Psi\left(K_{i_{-} \text {expect }}\right)$ is a nonlinear function. Thus, the general linear function such as $\Psi\left(K_{i_{-} \text {expect }}\right)=k \frac{K_{i_{\text {expect }}}}{K_{i_{-} \text {expect }(\max )}}+b$ can not meet the curve. Through experiments, the expressions of non-linear appraisal function is as follows:

$$
\Psi\left(K_{i_{-} \text {expect }}\right)=\frac{1}{2} \sin \left(\frac{\pi}{K_{i_{-} \text {expect }(\max )}} K_{i_{-} \text {expect }}-\frac{\pi}{2}\right)+\frac{1}{2}
$$

In this way, the threshold formula can be improved as:

$$
\begin{aligned}
T(n)= & \frac{p}{1-p \times[r \cdot \bmod (1 / p)]} \cdot \Psi\left(K_{i_{-} \text {expect }}\right) \\
= & \frac{p}{1-p \times[r \cdot \bmod (1 / p)]} \\
& \cdot \frac{1}{2} \sin \left(\frac{\pi}{K_{i_{-} \text {expect(max) }}} K_{i_{-} \text {expect }}-\frac{\pi}{2}\right)+\frac{1}{2}
\end{aligned}
$$

The optimal numbers of cluster-head nodes of this article use the method of literature [16], as shown in (9).

$$
k_{\text {opt }}=\frac{\sqrt{N}}{\sqrt{2 \pi}} \sqrt{\frac{\varepsilon_{f s}}{\varepsilon_{m p}}} \cdot \frac{M}{d_{\text {toBS }}^{2}} E_{\text {elec }} / p J \cdot\left(\text { bit } \cdot m^{-2}\right)^{-1} m^{2}
$$

where $k_{\text {opt }}$ denotes the optimal numbers of cluster-head nodes, $N$ denotes the total sensor nodes, $M$ denotes the side of the node distribution area and $d_{t o B S}$ denotes the distance from the node to the base station.

When the distribution of the energy of sensor node was extremely unbalanced, the modified threshold formula (8) can avoid the tiny alteration of $T(n)$. Since the nodes with high energy would have more probability to become the cluster-heads than the one with low energy, it also reduces the randomness of cluster selection in network. The mechanism can balance the energy consumption among the nodes and prolong the lifetime of the whole network. When the energy of cluster-heads is well-balanced, the value of cluster-head node expected frequency appraisal function is equal to one approximately.

\subsection{Steady-State Phase}

In the steady-state phase, each node sends the collected information during its own TDMA time slot [17]. After receiving information of all the cluster-heads, the base station analysis the datum and transfer those to the top man-machine communication interface. According to the
ID and information intensity of node sending, the cluster-heads broadcast the information to the network, and prepare for the next round.

So far, this round is complete. Figure $\mathbf{3}$ is a flowchart of the improved algorithm.

\section{Simulation Results}

In this section, we mainly use simulations to analyze and evaluate the performance of the algorithm. This paper uses the network simulator, NS2 [18], which developed by UC Berkeley, to simulate the method. Then, to verify the improved algorithm proposed in this paper, we will compare the results with LEACH and LEACH-C.

\subsection{Simulation Setup}

As shown in Figure 4, 100 sensor nodes were arranged randomly in the field of $100 \times 100$ square meters. The sink nodes were also arranged. The nodes have no movement capability, and they can correspond with the sink nodes directly. The initial energy of a sensor node is $2 \mathrm{~J}$ and the energy of a sink node is infinite. The data information of the next round can not be sent until the end of the former transmitted. It is assumed that the consumption of the energy can be ignored in the execution. The settings of other parameters of the network environment were displayed in Table 1.

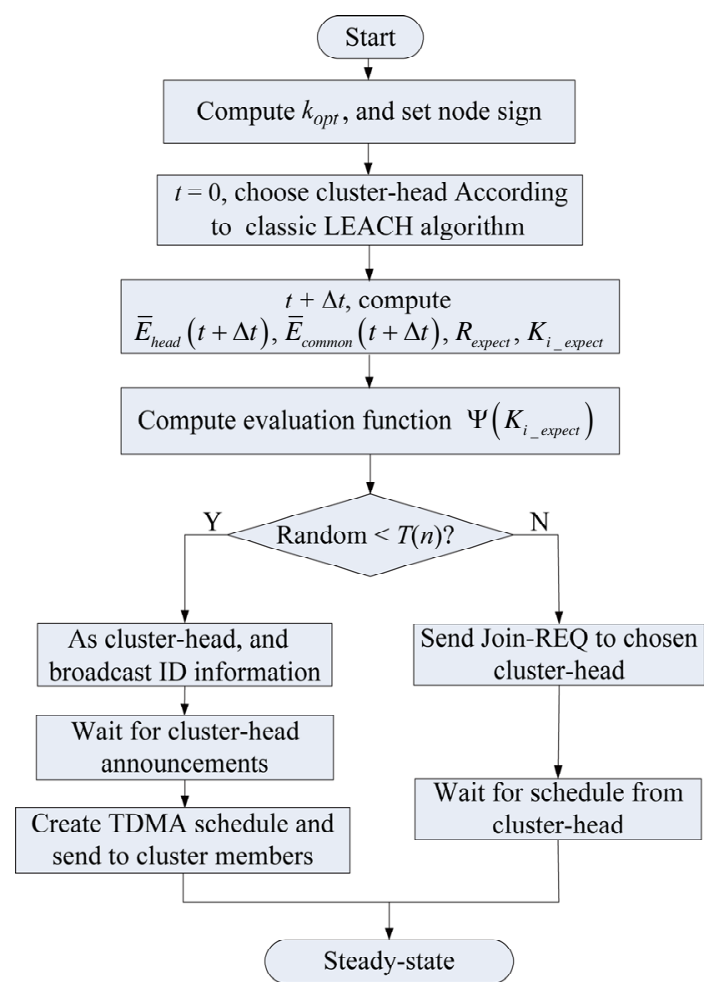

Figure 3. Flowchart of LEACH-HEFA. 


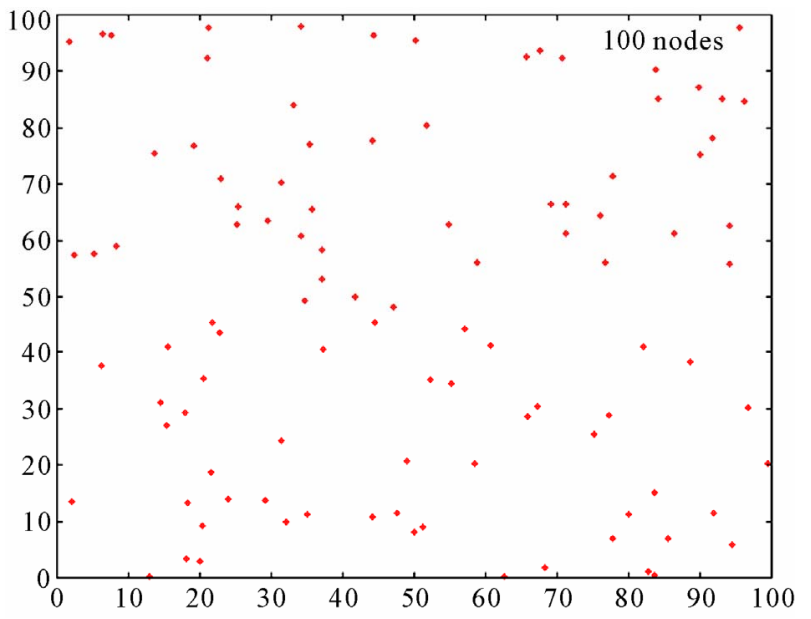

Figure 4. Random deployment of sensor nodes in the field.

Table 1. Simulation parameters.

\begin{tabular}{ll}
\hline \multicolumn{1}{c}{ Parameters } & \multicolumn{1}{c}{ Value } \\
\hline Number of nodes & 100 \\
Network size & $100 \times 100 \mathrm{~m}^{2}$ \\
Distance of node transmission & $20 \mathrm{~m}$ \\
Initial energy & $2 \mathrm{~J}$ \\
$E_{\text {elec }} / n J \cdot b i t^{-1}$ & 50 \\
$\left(\varepsilon_{f s}\right) / p J \cdot\left(\text { bit } \cdot \mathrm{m}^{-2}\right)^{-1}$ & 10 \\
$\left(\varepsilon_{m p}\right) / p J \cdot\left(\text { bit } \cdot \mathrm{m}^{-2}\right)^{-1}$ & 0.0013 \\
Expected number of cluster-head per round & 5 \\
\hline
\end{tabular}

\subsection{Simulations and Analysis}

Figure 5 shows the number of nodes alive over time of the three algorithms. As shown in this figure, compared with the LEACH and LEACH-C algorithms, the lifetime of the sensor node was prolonged after the improvement. The LEACH-HEFA algorithms optimized the clusterhead selection mechanism by proposing the cluster-head node expected frequency appraisal function. Thus it makes the node premature deceasing because of the excessive energy consumption, and then can prolong the network lifetime.

Figure 6 shows the curve of the total energy consumption of the network node varied with the time. The top curve presents the energy consumption in LEACH algorithms. The lifetime of the whole network is short because of the frequent cluster-head selection without considering the balance of energy. After the improvement of the cluster-head selection mechanism, the nodes with lower energy become the cluster-head with fewer probabilities, and the cluster-head would not vary frequently. Compared with the LEACH and LEACH_C algorithms under the same runtime, the energy consump- tion of the whole network would decrease remarkably.

As shown in Figure 7, the network operated regularly to 200 rounds, all of the water-environment sensor nodes can transmit the data normally. The first node is already dead when the runtime of network operate to 200 and 240 rounds in the LEACH and LEACH_C algorithms respectively. However, after the improvement, the first node would not die until the 290 round because of the energy consumption. The runtime of the last node is prolonged significantly. Compared with the LEACH and LEACH-C algorithms, the results show that the network lifetime of the improvement algorithm can be prolonged by $23 \%$ and $18 \%$, respectively.

\section{Conclusions}

The cluster-head selection mechanism and energy saving strategy play an important role in the design of network routing protocol. However, the current routing protocols usually have problems such as previously selected cluster-head, unbalancing energy loads and short lifetime of

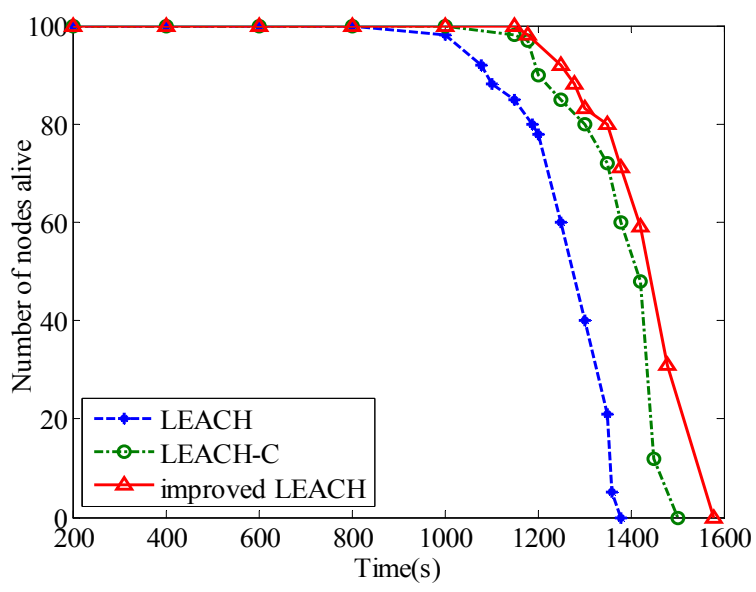

Figure 5. Number of nodes alive over time.

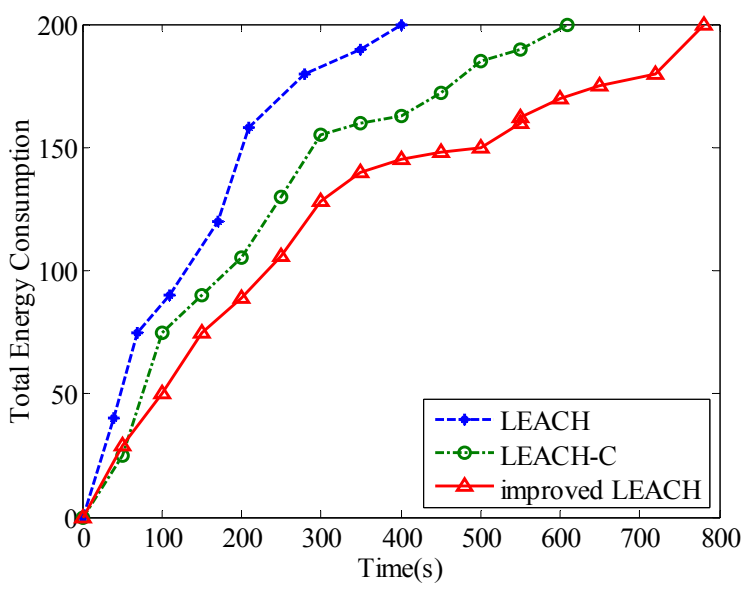

Figure 6. Total energy consumption versus time. 


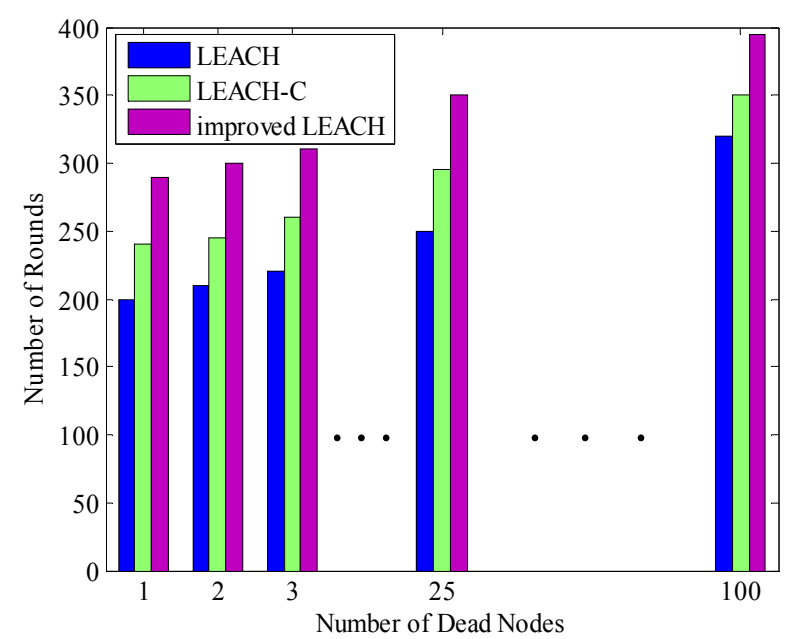

Figure 7. The number of rounds and dead nodes of three algorithms.

network. Therefore, it can not be applied to the waterenvironment monitoring network, which is real-time system with a large range to collect, transmit, analyze and process water-environment parameters. In this paper, an improved LEACH algorithm, LEACH-HEFA (LEACHHead Expected Frequency Appraisal) algorithm is proposed based on the idea of cluster-head expected frequency appraisal. If the node has higher residual energy, it will have more probability to be selected as clusterhead, which balances node energy effectively. Simulation results show that LEACH-HEFA algorithm is suitable to the WMN. It also can equalize the energy consumption of the nodes in the network and prolong the network lifetime significantly.

\section{References}

[1] L. Fl, "Wireless Sensor Networks," Smart Environments: Technologies, Protocols, and Applications, Vol. 21, No. 2, 2004, pp. 1-18.

[2] P. Corke and T. Wark, "Environmental Wireless Sensor Networks," Proceedings of the IEEE, Vol. 98, No. 11, 2010, pp. 1903-1917. doi:10.1109/JPROC.2010.2068530

[3] X. J. Yan, L. M. Lu and L. Z. Xu, "The Application of Wireless Sensor Network in the Irrigation Area Automatic System," International Conference on Networks Security, Wireless Communications and Trusted Computing, Vol. 2, 25-26 April 2009, pp. 21-24.

[4] T. H. Fan, L. Z. Xu, X. W. Zhang and H. B. Wang, "Research and Design of a Node of Wireless Multimedia Sensor Network," Proceedings of the 5th International Conference on Wireless Communications, Networking and Mobile Computing, Piscataway, 24-26 September 2009.

[5] S. Guo, H. Zhang and H. Chen, "A Reservoir Flood Forecasting and Control System for China," Hydrological Sciences Journal, Vol. 49, No. 6, 2006, pp. 959-972.
[6] X. F. Li, L. Z. Xu and H. B. Wang, "A Differential Evolution-Based Routing Algorithm for Environmental Monitoring Wireless Sensor Networks," Sensors, Vol. 10, No. 6, 2010, pp. 5425-5442.

[7] H. B. Wang, Y. L. Zhong, J. H. Zhang, X. J. Yan, L. Z. $\mathrm{Xu}$, "Based on Sleep Scheduling for Wireless Sensor Networks Clustering Protocol," Microelectronics \& Computer, Vol. 27, No. 8, 2010, pp. 226-229.

[8] O. Younis and S. Fahmy, "HEED: A Hybrid, Energy Efficient, Distributed Clustering Approach for Ad-Hoc Sensor Networks," IEEE Transactions on Mobile Computing, Vol. 3, No. 4, 2004, pp. 660-669. doi:10.1109/TMC.2004.41

[9] A. B. M. A. Al Islam, C. S. Hyder, H. Kabir and M. Naznin, "Finding the Optimal Percentage of Cluster Heads from a New and Complete Mathematical Model on LEACH," Wireless Sensor Network, Vol. 2, No. 2, 2010, pp. 129-140.

[10] J. Zhu, J. Li and H. Gao, "Tasks Allocation for RealTime Applications in Heterogeneous Sensor Networks for Energy Minimization," 8th ACIS International Conference on Software Engineering, Artificial Intelligence, Networking, and Parallel/Distributed Computing (SNPD 2007), Qingdao, 30 July-1 August 2007, pp. 20-25.

[11] G. Anastasi, M. Conti, M. Di Francesco and A. Passarella, "Energy Conservation in Wireless Sensor Networks," A Survey, Ad Hoc Networks, Vol. 7, No. 3, 2009, pp. 537568. doi:10.1016/j.adhoc.2008.06.003

[12] P. Tillapart, T. Thumthawatworn and P. Pakdeepinit, "Method for Cluster Heads Select Ion in Wireless Sensor Network," Proceedings of the 2004 IEEE Aerospace Conference, IEEE, Piscataway, 6-13 March 2004, pp. 3615-3623.

[13] B. Nazir and H. Hasbullah, "Energy Balanced Clustering in Wireless Sensor Network," 4th International Symposium on Information Technology 2010 (ITSim'10), Kuala Lumpur, 15-17 June 2010.

[14] N. M. A. Latiff, C. Tsimenidis and B. S. Sharif, "Performance Comparison of Optimization Algorithms for Clustering in Wireless Sensor Networks," MASS, Pisa, 8-11 October 2007, pp. 1-4.

[15] A. Manjeshwar and D. P. Grawal, "TEEN: A Protocol for Enhanced Efficiency in Wireless Sensor Networks," Proceedings of the 15th Parallel and Distributed Processing Symposium, IEEE Computer Society, San Francisco, 23-27 April 2001.

[16] Y. Tao and Y. L. Zheng, "The Combination of the Optimal Number of Cluster-Heads and Energy Adaptive Cluster-Head Selection Algorithm in Wireless Sensor Networks," WiCOM, International Conference, Wuhan, 23-25 September 2011, pp. 1-4.

[17] E. W. Heinz, "Application Specific Protocol Architectures for Wireless Networks," Massachusetts Institute of Technology, Boston, 2000.

[18] T. Issariyakul and E. Hossain, "Introduction to Network Simulator NS2," Springer, Berlin, 2008. 